\title{
Dynamic optimization analysis of hydraulic pipeline system based on a developed response surface method
}

\author{
Hongquan $\mathrm{QU}^{1}$, Jianlin SUN ${ }^{1}$, Xu YAN ${ }^{1}$, Yuanlin ZHANG $^{1}$, Xuefeng LIU ${ }^{1}$, Tao YU $^{1^{*}}$, Huawei \\ $\mathrm{HAN}^{2}$, Langjun $\mathrm{XU}^{2}$
}

1 School of Electromechanical and Automotive Engineering, Yantai University, Yantai 264005, China

2 Yantai CIMC Raffles Offshore Limited, Yantai 264670, China

*Corresponding Author: Tao YU, Corresponding address, 30, Qingquan RD, Laishan District, Yantai, China; email address: yutao@ytu. edu.cn

\begin{abstract}
:
When designing a complex pipeline with long distance and multi-supports for offshore platform, it is necessary to analyze the vibration characteristics of the complex pipeline system to ensure that there is no harmful resonance in the working conditions. Therefore, the optimal layout of support is an effective method to reduce the vibration response of hydraulic pipeline system. In this paper, a developed dynamic optimization method for the complex pipeline is proposed to investigate the vibration characteristics of complex pipeline with multi-elastic supports. In this method, the Kriging response surface model between the support position and pipeline is established. The position of the clamp in the model is parameterized and the optimal solution of performance index is obtained by genetic algorithm. The number of clamps and the interval between clamps are considered as the constraints of layout optimization, and the optimization objective is the natural frequencies of pipeline. Taking a typical offshore pipeline as example to demonstrate the effectiveness of the proposed method, the results show that the vibration performance of the hydraulic pipeline system is distinctly improved by the optimization procedure, which can provide reasonable guidance for the design of complex hydraulic pipeline system.
\end{abstract}

Keywords: Hydraulic pipeline; Multi-Support; Response surface method; Optimization analysis

\section{Introduction}

The hydraulic pipeline system is usually employed to transmit power in drilling platform. It has the characteristics of complex configuration, long span and multi-supports. When the pipeline is excited by external mechanical load and internal fluid, the large vibration of the pipeline system occurs, which will directly lead to fatigue failure, displacement deformation, loosening of clamp and fatigue fracture of connecting parts. The design of offshore platform pipeline is of great significance to maintain productivity operation and ensure the safety of the platform. With the deepening of drilling depth, the vibration problem of pipeline system becomes more prominent. The vibration analysis of pipeline is of great significance to the layout of pipeline and the optimization of supports.

The investigation for the dynamic optimization characteristics of pipeline has received extensive attention in recent years. For example, Kwong et al. ${ }^{[1]}$ proposed a novel genetic algorithm to optimize the support position for reducing vibration in hydraulic systems. A simple hydraulic rig was conducted as an example. Numerical and experimental results showed that the significant reductions in vibration was obtained by the optimal support location. Wang et al. ${ }^{[2-3]}$ presented an evolutionary shift method to solve optimization of support positions with a fixed grid mesh, three numerical examples were conducted to demonstrate the effectiveness of the optimization method. In order to avoid dangerous forced vibrations or resonance, the clamp numbers and locations of a pipejoint system were optimized by Liu et al ${ }^{[4]}$. The results showed that the optimized pipe-joint system has better anti-vibration ability. Li et al. ${ }^{[5]}$ presented a chaotic swarm particle optimization algorithm to determine optimal clamp locations for hydraulic pipelines in aircraft. Results showed that the value of objective function was decreased by $77.67 \%$ compared to the original system by optimal clamp locations. The global sensitivity analysis based on the Sobol method was presented to determine the influence of clamp position on the natural frequency of pipeline system. The simulation and experiment results showed that the proposed optimization method can reduce the vibration of the pipeline system significantly. ${ }^{[6]}$ 
From the reviewed references, the current optimization method is time-consuming for the global optimal solution of long span pipeline. A popular approach for global optimization is proposed in which the simulation model generates data for the construction of simpler model as a source of computer experiment. The early research about the response surface method is proposed to improve the calculation optimization efficiency ${ }^{[7]}$. In recent years, the response surface method has been applied in many fields ${ }^{[8-20]}$. For example, Xiao et al. ${ }^{[8]}$ proposed response surface method to study the effects of the different content of $\mathrm{Si}_{3} \mathrm{~N}_{4}$ particles in the plating solution. Zhang et al. ${ }^{[9]}$ presented the response surface method to solve the problem of slope stability. Xue et al. ${ }^{[16]}$ proposed the improved response surface method to solve the various related deck landing parameters on sinking velocity for carrier-based aircraft. In order to improve the calculation efficiency of the vibration characteristics of turbine blade disharmony, an improved substructure mode synthesis method based on the extreme response surface method is proposed by Bai et al. ${ }^{[18]}$. Su et al. ${ }^{[19]}$ proposed a response surface method to study the fatigue reliability for metal dual inline packages under random vibration. Considering the integrality and locality of long span and multi-elastic support of pipelines on offshore platforms, the response surface method can be used to optimize the support locations for hydraulic pipeline system.

To the author's best knowledge, there is almost no literature reporting on the response surface method for the design optimization of hydraulic pipelines with support. As a promising global optimization method, it is the first time to investigate the dynamic optimization analysis of hydraulic pipeline system with multi-supports. Firstly, the method of mathematical statistics combined with pipeline dynamics analysis was adopted to analyze the influence of the clamps layout. Then the developed response surface method is used to establish the optimization model. By simulating the implicit relation between variables and performance indexes, the optimal solution of performance indexes is obtained by genetic algorithm. Taking a typical offshore pipeline as an example to demonstrate the effectiveness of the presented method, an optimization procedure for pipeline is conducted. The dynamic optimization process for complex pipeline with multi-elastic supports are discussed further.

\section{Optimization method}

The mathematical equation of design variables and response quantities can be expressed as:

$g(x)=y+\varepsilon=f\left(x_{1}, x_{2}, x_{3} \cdots x_{n}\right)+\varepsilon$

where $\mathrm{g}(\mathrm{x})$ is the amount of response, $\mathrm{y}$ is the function of objective and $\varepsilon$ is the fitting error between $\mathrm{g}(\mathrm{x})$ and $\mathrm{y}$.

The multi-level function relations can be written as:

$\tilde{y}=\alpha_{0}+\sum_{j=1}^{n} \alpha_{j} x_{j}+\sum_{j=n+1}^{2 n} \alpha_{j} x_{j-k}^{2}+\sum_{i=1}^{n-1} \sum_{j=i+1}^{n} \alpha_{i j} x_{i} x_{j}$

where $\mathrm{n}$ is the number of design variable, $\alpha_{0}$ is the undetermined constant term, $a_{j}$ is the undetermined coefficient of the linear term, and $\alpha_{i j}$ is the undetermined coefficient of the quadratic term.

By variable transformation, the equation (2) can be expressed as:

$$
\begin{gathered}
\left\{\begin{array}{c}
x_{0}=1 \\
x_{1}=x_{1}, x_{2}=x_{2}, \cdots x_{n}=x_{n} \\
x_{n+1}=x_{1}^{2}, x_{n+2}=x_{2}^{2}, \cdots x_{2 n}=x_{n}^{2} \\
x_{2 n+1}=x_{1}, x_{2 n+1}=x_{1}, \cdots x_{k-1}=x_{n-1} x_{n} \\
\beta_{0}=\alpha_{0} \\
\beta_{1}=\alpha_{1}, \beta_{2}=\alpha_{2}, \cdots \beta_{n}=\alpha_{n} \\
\beta_{n+1}=\alpha_{n+1}, \beta_{n+2}=\alpha_{n+2}, \cdots \beta_{2 n}=\alpha_{2 n} \\
\beta_{2 n+1}=\alpha_{12}, \beta_{2 n+2}=\alpha_{13}, \cdots \beta_{k-1}=\alpha_{(n-1) n}
\end{array}\right.
\end{gathered}
$$

By substituting equation (3) and equation (4) into equation (2), it can be obtained:

$\tilde{y}=\beta_{0}+\sum_{i=1}^{k-1} \beta_{i} \alpha_{i}$

The response of the corresponding matrix can be expressed as:

$$
\left[\begin{array}{c}
y_{1} \\
y_{2} \\
\vdots \\
y_{n}
\end{array}\right]=\left[\begin{array}{ccccc}
1 & x_{11} & x_{21} & \cdots & x_{n 1} \\
1 & x_{12} & x_{22} & \cdots & x_{n 2} \\
\vdots & \vdots & \vdots & \cdots & \cdots \\
1 & x_{1 k} & x_{2 k} & \cdots & x_{n k}
\end{array}\right]\left[\begin{array}{c}
\beta_{0} \\
\beta_{1} \\
\vdots \\
\beta_{n}
\end{array}\right]+\left[\begin{array}{c}
\varepsilon_{0} \\
\varepsilon_{1} \\
\vdots \\
\varepsilon_{n}
\end{array}\right]
$$

In order to obtain the response surface with the smallest error. The least-square method is used which can be expressed as:

$$
s(\beta)=\sum_{j=1}^{m} \varepsilon_{j}^{2}=\sum_{j=1}^{m}\left(\sum_{i=0}^{k-1} \beta_{i} x_{i}^{(j)}-y^{(j)}\right)^{2}
$$

The minimum value can be expressed as:

$$
\frac{\partial S}{\partial \beta_{i}}=2 \sum_{j=1}^{m}\left(x_{i}^{(j)}\left(\sum_{i=0}^{k-1} \beta_{i} \alpha_{i}-y^{(j)}\right)\right)=0(l=0, \cdots, k-1)
$$

The equation (8) can be written as the matrix form:

$$
(X \beta-y)^{T} X=0
$$

The response surface model can be obtained by calculating $\beta$.

The satisfaction function of MOGA genetic algorithm is usually used to optimize multiple objectives. The multiobjective optimization is to find the compromise optimal solution by considering each objective synthetically in the constraint region. A multi-objective optimization problem without losing generality has $\mathrm{n}$ decision variables and $\mathrm{m}$ objective variables which can be expressed as:

$$
\left\{\begin{array}{c}
\min y=F(x)=\left\{f_{1}(x), f_{2}(x), f_{3}(x), \cdots, f_{m}(x)\right\} \\
\text { s.t. } g_{i}(x) \leq 0, i=1,2, \cdots, l \\
h_{j(x)}=0, j=1,2, \cdots, k
\end{array}\right.
$$

Where $\mathrm{x}$ is $\mathrm{n}$-dimensional decision vector, $\mathrm{y}=\mathrm{F}(\mathrm{x})$ is the target vector of $\mathrm{m}$ dimension. $\mathrm{l}$ is the constraints of inequalities, $\mathrm{k}$ is equality constraints.

Pareto optimal solution can be defined as Ps. The surface composed of the target vectors corresponding to all Pareto optimal solutions can be written as $\mathrm{P}_{\mathrm{F}}$ : $P_{F}=\left\{F\left(x^{*}\right)=\left(f_{1}\left(x^{*}\right), f_{2}\left(x^{*}\right), f_{3}\left(x^{*}\right), \cdots, f_{m}\left(x^{*}\right)\right)^{T} \mid x^{*} \in P_{S}\right\}$ 
In order obtain the real surface, Box-Behnken design (BBD) scheme is the most potent screening design method in response surface optimization. In this method, the selecting points from the three-level factorial arrangement can obtain the efficient estimation of coefficients of the mathematical model. The distribution of three-level full factorial design is illustrated in figure 1.

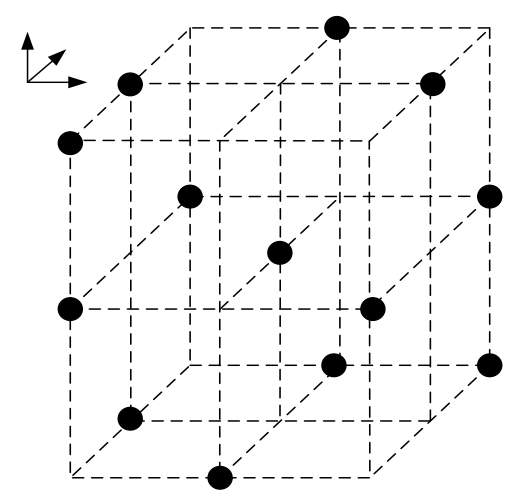

Figure 1 Distribution of three-level full factorial design ${ }^{[20]}$

\section{Design and optimization of response surface method}

For the optimizing support locations of pipeline system, the optimization objective should be determined firstly. The pump fluid fluctuation can induce serious pipeline vibration and cause pipeline failure. The pump fluid fluctuation frequency is $275 \mathrm{~Hz}$ by test in offshore platform. So the natural frequency of pipeline should be far away from the excited frequency $275 \mathrm{~Hz}$. Therefore, in order to avoid the pipeline mechanical resonance, the optimization objective is to avoid the resonance of the natural frequency of the pipeline system.

As shown in figure 2. The pipeline system consists three clamps, the vibration characteristics of pipeline has been analyzed, and the modal shapes have been obtained. The parameterization of pipeline system is shown in Table 1.

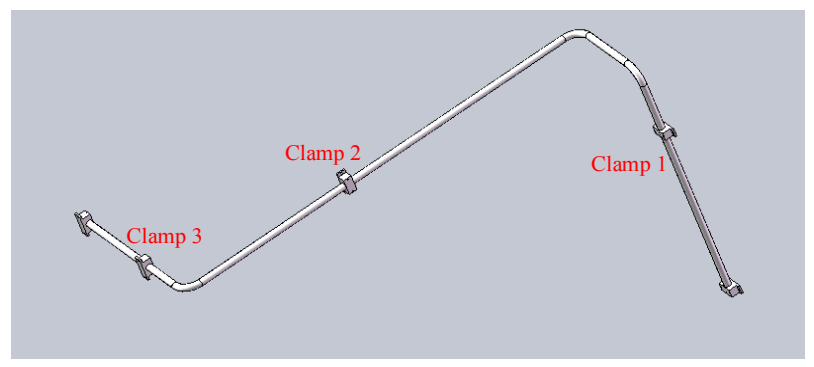

Figure 2 Diagram of piping system

Table 1 Parameterization of pipeline system

\begin{tabular}{ccc}
\hline size & Numerical value & Estimate \\
\hline DS_D1@distance 1 & $1200 \mathrm{~mm}$ & $1200 \mathrm{~mm}$ \\
DS_D1@distance 2 & $1500 \mathrm{~mm}$ & $1500 \mathrm{~mm}$ \\
DS_D1@distance 3 & $400 \mathrm{~mm}$ & $400 \mathrm{~mm}$ \\
\hline
\end{tabular}

In order to optimize the clamp position of the pipeline system, the modal analysis for the pipeline system has been conducted to obtain the natural frequency of the pipeline system. The geometric parameter of the selected pipeline is shown in Table 2.

Table 2 Parameter of the pipeline

\begin{tabular}{cccc}
\hline Designation & Symbol & Unit & Numerical \\
\hline Elastic modulus & $\mathrm{E}$ & $\mathrm{Pa}$ & $2.06 \times 1011$ \\
Density & $\rho$ & $\mathrm{kg} / \mathrm{m}^{3}$ & $7.9 \times 103$ \\
Poisson's ratio & $\mu$ & - & 0.3 \\
Total length of pipeline & $\mathrm{l}$ & $\mathrm{m}$ & 4.5 \\
outer diameter & $\mathrm{r} 1$ & $\mathrm{~m}$ & 0.04 \\
inner diameter & $\mathrm{r} 2$ & $\mathrm{~m}$ & 0.036 \\
\hline
\end{tabular}

As shown in Figure 3 . The $10^{\text {th }}$ order natural frequency of the pipeline is $254.9 \mathrm{~Hz}$ and the $11^{\text {th }}$ order natural frequency of the pipeline is $285.58 \mathrm{~Hz}$. The test design table is obtained by the $\mathrm{BBD}$ scheme, respectively.

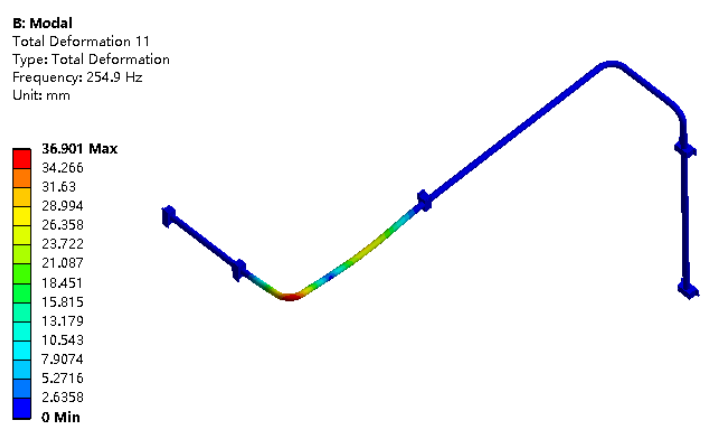

(a) $10^{\text {th }}$ order natural frequency

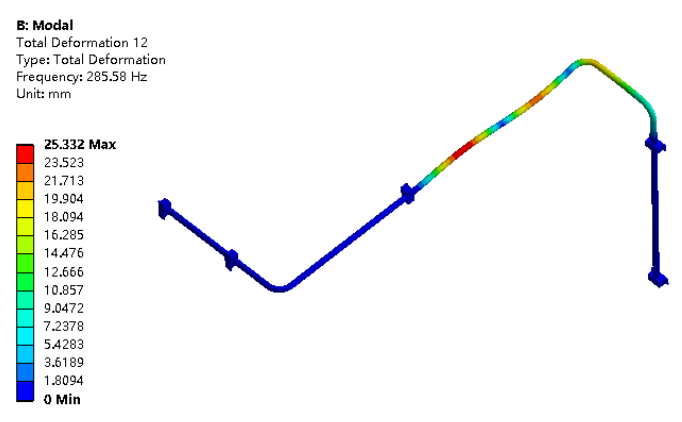

(b) $11^{\text {th }}$ order natural frequency

Figure 3 Natural frequencies and mode shapes of pipeline system

The optimization process of pipeline system is shown in figure 4 . As can be seen from figure 4 . The parametric modeling should be established firstly, it consists of the variable scope, test design and sample point. Then the dynamic analysis of the pipeline has been conducted and the optimization objective can be obtained. The test design table can be established and the response surface model can be obtained. Finally, optimal solution for pipeline supports can be obtained by optimizing the parameters. 


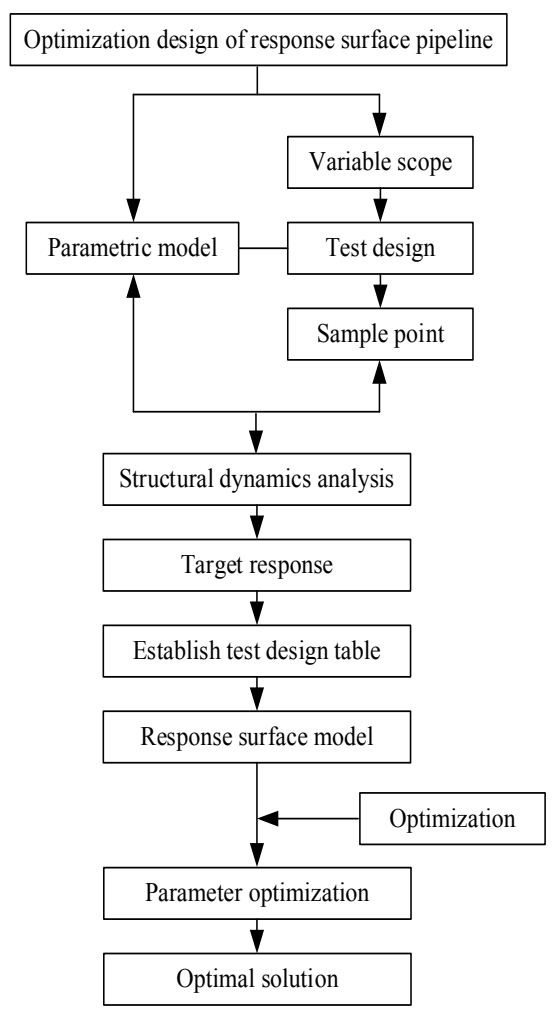

Figure 4 Optimization flow chart of response surface method

\section{Test design table and response surface modelling}

\subsection{Test design table}

In this section, the natural frequencies of pipeline are defined as the optimized response value, the Kriging response surface model of the clamp position is established, and the optimization position of the clamp position is obtained by the candidate points. The position of the clamp in the model is parameterized in the modeling software, and the design variables are shown in figure 5 . The model is imported into the finite element analysis software, and modal analysis has been conducted. The maximum deformation is taken as the response target value. Using the BBD scheme, the change intervals of design variables are shown in Table 1, and the combination relationship of design variables are shown in Table 3 and Table 4, respectively.

According to the dynamic analysis of the design variable data listed in Table 2 and Table 3, the numerical change of the natural frequency of the pipeline can be obtained. The results are shown in Table 5. The P4 and P5 values in Table 5 are the basis parameters for establishing the response surface model.

Table 3 Change range of BBD variable

\begin{tabular}{lccc}
\hline & Lower limit & Upper limit & Original size \\
\hline distance $1 / \mathrm{mm}$ & 1080 & 1320 & 1200 \\
distance $2 / \mathrm{mm}$ & 1350 & 1650 & 1500 \\
distance $3 / \mathrm{mm}$ & 360 & 440 & 400 \\
\hline
\end{tabular}

Table 4 BBD test plan

\begin{tabular}{ccccc}
\hline 1 & Test number & P1-distance 1 & P2-distance 2 & P3- distance 3 \\
\hline 2 & 1 & 1200 & 1500 & 400 \\
3 & 2 & 1080 & 1500 & 360 \\
4 & 3 & 1320 & 1500 & 360 \\
5 & 4 & 1080 & 1500 & 440 \\
6 & 5 & 1320 & 1500 & 440 \\
7 & 6 & 1080 & 1350 & 400 \\
8 & 7 & 1320 & 1350 & 400 \\
9 & 8 & 1080 & 1650 & 400 \\
10 & 9 & 1320 & 1650 & 400 \\
11 & 10 & 1200 & 1350 & 360 \\
12 & 11 & 1200 & 1350 & 440 \\
13 & 12 & 1200 & 1650 & 360 \\
14 & 13 & 1200 & 1650 & 440 \\
\hline
\end{tabular}

Table 5 BBD test design table

\begin{tabular}{|c|c|c|c|c|c|c|}
\hline 1 & Test number & P1-distance1 & P2- distance2 & P3- distance 3 & P4- $10^{\text {th }}$ fixed frequency & P5- $11^{\text {th }}$ fixed frequency \\
\hline 2 & 1 & 1200 & 1500 & 400 & 254.9 & 285.58 \\
\hline 3 & 2 & 1080 & 1500 & 360 & 277.29 & 278.65 \\
\hline 4 & 3 & 1320 & 1500 & 360 & 277.25 & 352.54 \\
\hline 5 & 4 & 1080 & 1500 & 440 & 242.32 & 277.83 \\
\hline 6 & 5 & 1320 & 1500 & 440 & 268.20 & 349.91 \\
\hline 7 & 6 & 1080 & 1350 & 400 & 215.54 & 329.92 \\
\hline 8 & 7 & 1320 & 1350 & 400 & 311.28 & 363.11 \\
\hline 9 & 8 & 1080 & 1650 & 400 & 242.57 & 244.68 \\
\hline 10 & 9 & 1320 & 1650 & 400 & 315.27 & 326.73 \\
\hline 11 & 10 & 1200 & 1350 & 360 & 238.02 & 335.80 \\
\hline 12 & 11 & 1200 & 1350 & 440 & 237.56 & 337.58 \\
\hline 13 & 12 & 1200 & 1650 & 360 & 248.80 & 272.46 \\
\hline 14 & 13 & 1200 & 1650 & 440 & 247.60 & 272.25 \\
\hline
\end{tabular}



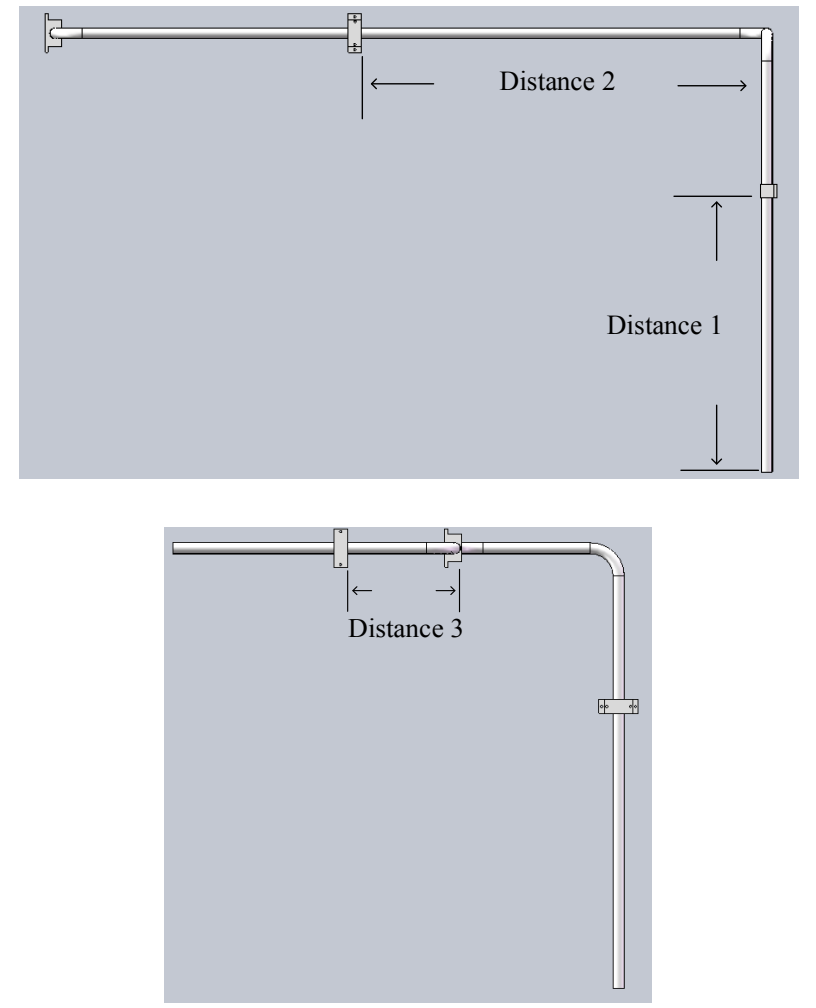

Figure 5 Design variables

\subsection{Response surface modeling}

According to the experimental design table, the response surface model type of the BBD scheme is defined as Kriging response surface. The Kriging method is used to solve the problem of error estimation. It is a spatial estimation technique of interpolation algorithm, and the fitting degree the sample points of the Kriging response surface and the predicted straight line is shown in figure 6 . The real values of P4 and P5 can match closely with the predicted values, and the established response surface model can meet the optimization requirement.

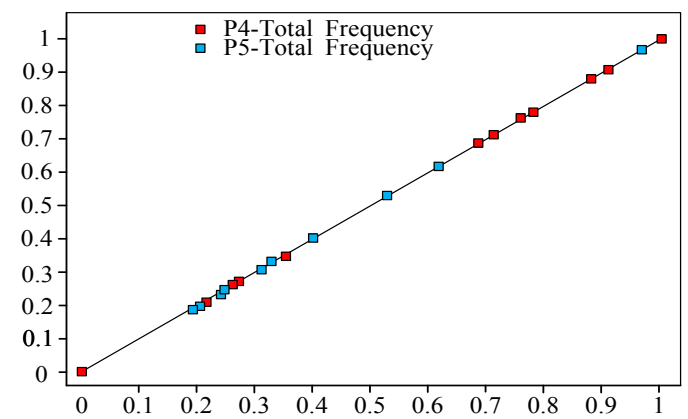

Figure 6 The fitting degree of the sample points of the Kriging response surface and the predicted straight line

The response surface model obtained by the BBD scheme is analyzed, as shown in figure 7. figure 7(a) is the response surface models of distance P1 and distance $\mathrm{P} 2$ with response target $\mathrm{P} 4$, figure $7(\mathrm{~b})$ is the distance P1 and distance P3 with response target P4, figure $7(\mathrm{c})$ is the distance P2 and distance P3 with response target P4.
Figure $7(\mathrm{~d})$ is the response surface models of distance P1 and distance P2 and response target P5. Figure 7 (e) is the distance $\mathrm{P} 1$ and distance $\mathrm{P} 3$ and response target $\mathrm{P} 5$. Figure 7 (f) is the distance P2 and distance P3 and response target P5.



(a) P1-P2-P4 response surface model

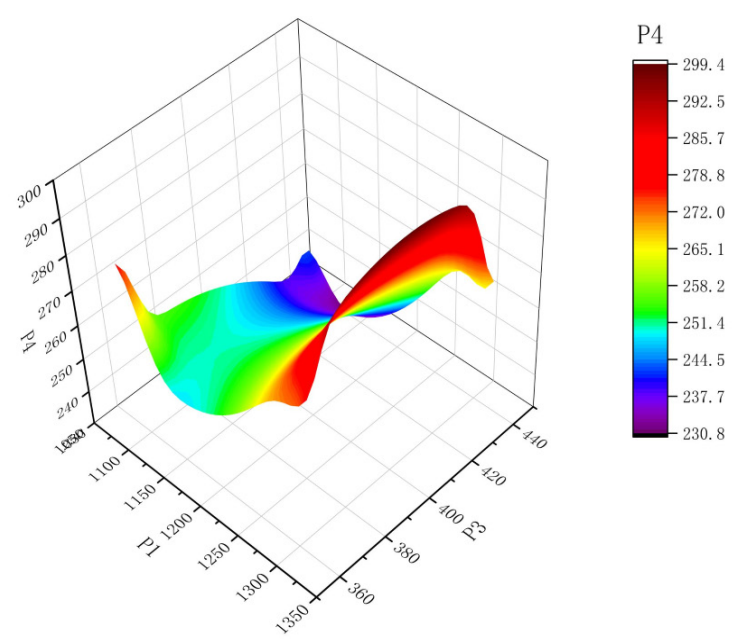

(b) P1-P3-P4 response surface model

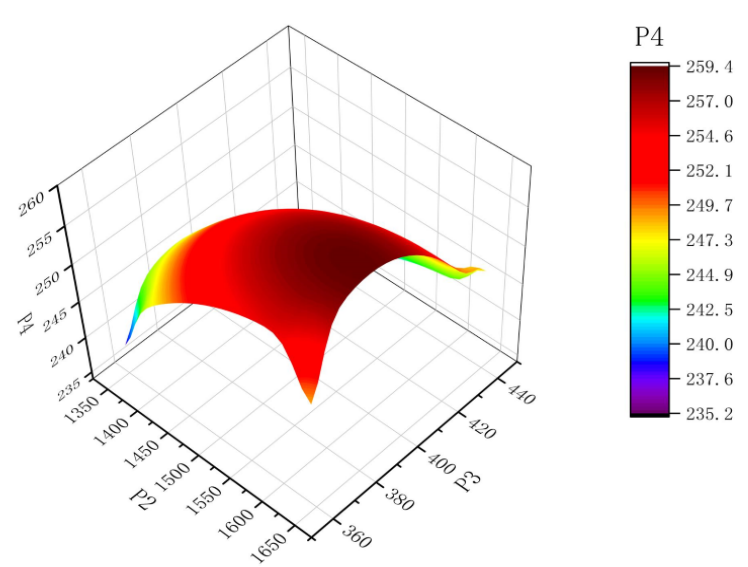

(c) P2-P3-P4 response surface model 


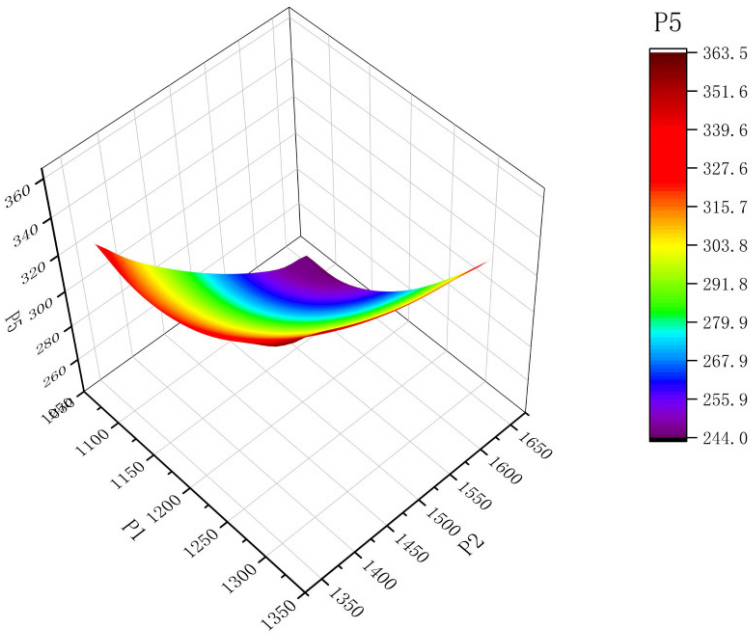

(d) P1-P2-P5 response surface model

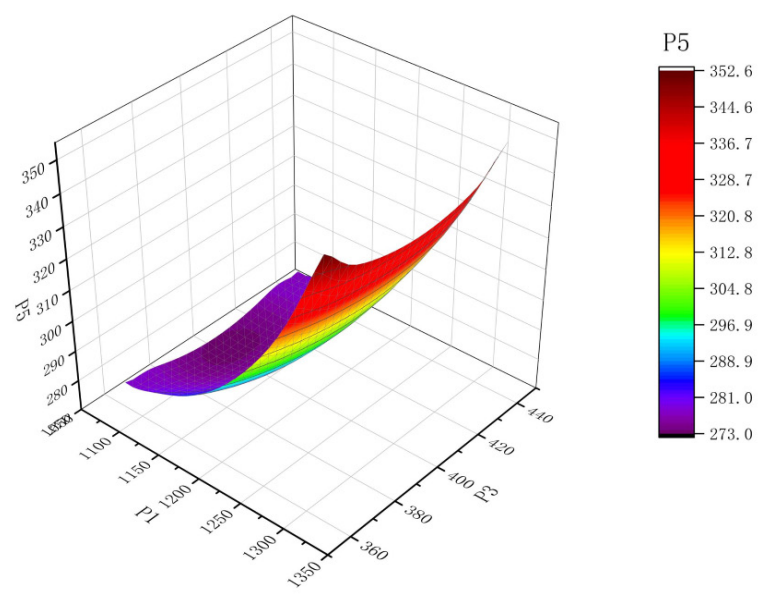

(e) P1-P3-P5 response surface model

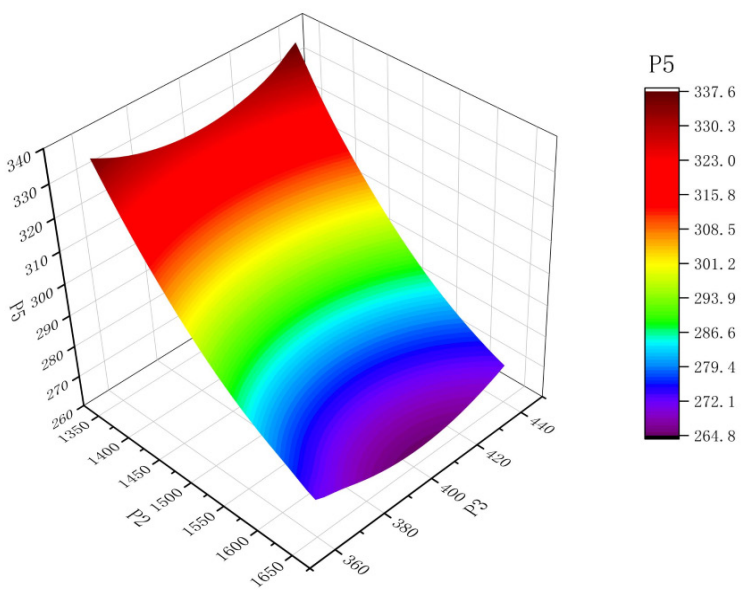

(f) P2-P3-P5 response surface model

Figure 7 Brig scheme Kriging response surface

From figure 7 , the design variables and the optimized target response values show obvious nonlinear characteristics. It can be seen that with the increasing of the distance P1, the optimization targets P4 and P5 increase slowly at first. When the distance P1 increases around 1200 , the increasing of P4 and P5 become faster, and the response surface model changes more drastically. When P2 gradually increases, the optimization target $\mathrm{P} 4$ increases rapidly and then slowly. When the distance P2 is around 1500 , the response surface model changes more seriously. The optimization target P5 gradually decreases with the increasing of P2. When P3 gradually increases to 400, the optimization target P4 increases slowly at first. When P3 is greater than 400 , the optimization target $\mathrm{P} 4$ decreases faster. The effect of P3 on P5 is gradually increased with the increasing of $\mathrm{P} 3$, and $\mathrm{P} 5$ decreases firstly and then increases with 400 as symmetry. In summary, the design variables and response values show complex nonlinear characteristics, and the response surface model can intuitively show the mutual relations.

\section{Results and discussions}

The effect of clamp position can change the natural frequencies of pipeline system. The pump excitation is $275 \mathrm{~Hz}$, when the natural frequency of the pipeline system are closer to pump excitation frequency, the mechanical resonance will occur. In order to avoid resonance, the natural frequency should be far away from $275 \mathrm{~Hz}$. Therefore, the optimization objective can reach the independent optimal value due to the interaction between design variables and the relationship between optimization objectives. The satisfaction function of MOGA genetic algorithm is usually used to optimize multiple objectives. The following definitions are given for the optimal solution in multi-objective optimization problems. The solution of the constraint conditions is defined as feasible solution. Pareto optimal solution is used as the optimal solutions. The surface composed of the target vectors corresponding to all Pareto optimal solutions. After MOGA analysis, it can be concluded that picture of Pareto front is shown in figure 8 , the blue dot value represents the best result.

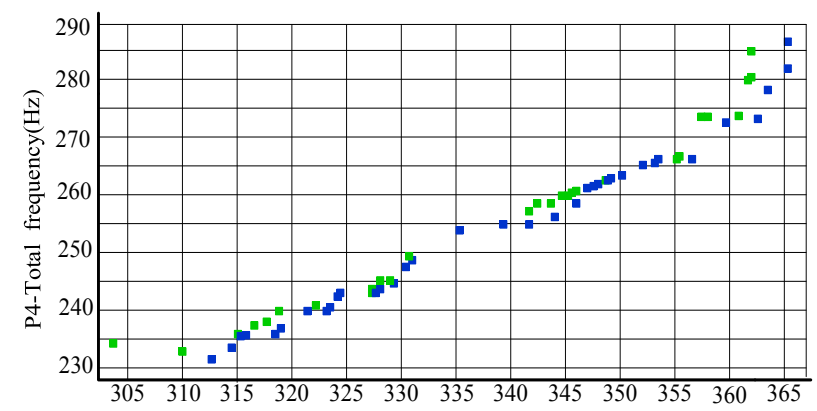

Figure 8 Pareto front

By Pareto front analysis, the optimal solution set can be obtained in which the blue point set is superior to the green point set. The candidate of BBD scheme can be obtained in the Kirging approximate response surface model, which are listed into Table 6. 
Table 6 BBD Candidate

\begin{tabular}{|c|c|c|c|}
\hline & Candidate 1 & Candidate 2 & Candidate 3 \\
\hline P1-Distance $1 / \mathrm{mm}$ & 1081.2 & 1201.1 & 1231.2 \\
\hline P2-Distance 2/mm & 1351.5 & 1351.3 & 1350.4 \\
\hline P3-Distance 3/mm & 439.15 & 439.09 & 438.22 \\
\hline $\mathrm{P} 4-10^{\text {th }}$ natural frequency $/ \mathrm{Hz}$ & 214.66 & 238.92 & 245.19 \\
\hline $\mathrm{P} 5-11^{\text {th }}$ natural frequency/Hz & 334.79 & 336.92 & 342.32 \\
\hline
\end{tabular}

As shown in the Table6, in order to avoid the mechanical resonance of the pipeline, the optimal values of each multi-objective optimization and the comprehensive optimal values of multi-objective optimization can be obtained, as shown in Table 7.

Table 7 Optimal candidate

\begin{tabular}{|c|c|c|c|c|c|}
\hline & P4 optimization & P5 optimization & $\begin{array}{c}\text { Comprehensive } \\
\text { optimization }\end{array}$ & $\begin{array}{c}\text { Before } \\
\text { optimization }\end{array}$ & $\begin{array}{c}\text { Difference } \\
\text { comparison }\end{array}$ \\
\hline P1-Distance $1 / \mathrm{mm}$ & 1081.2 & 1231.2 & 1081.2 & 1200.0 & 118.8 \\
\hline P2-Distance $2 / \mathrm{mm}$ & 1351.5 & 1350.4 & 1351.5 & 1500.0 & 148.5 \\
\hline P3-Distance $3 / \mathrm{mm}$ & 439.15 & 438.22 & 439.15 & 400.00 & 39.15 \\
\hline $\mathrm{P} 4-10^{\text {th }}$ natural frequency/Hz & 214.66 & 245.19 & 214.66 & 254.90 & 40.24 \\
\hline $\mathrm{P} 5-11^{\text {th }}$ natural frequency $/ \mathrm{Hz}$ & 334.79 & 342.32 & 334.79 & 285.58 & 49.21 \\
\hline
\end{tabular}

It can be seen from the Table 7 that the natural frequencies of pipeline system can be changed significantly by multi-objective optimization. If the response value is analyzed separately, different optimal design variables will be obtained. However, it is necessary to select a combination of design variables which are different from the natural frequency of the pipeline system and the pump fluid excitation frequency. The optimization objective is that the natural frequency of pipeline should be far away from the excited frequency $275 \mathrm{~Hz}$. Therefore, P1 $=1081.2 \mathrm{~mm}, \mathrm{P} 2$ $=1351.5 \mathrm{~mm}, \mathrm{P} 3=439.15 \mathrm{~mm}$ are the best combination of design variables. Moreover, the $10^{\text {th }}$ natural frequency of pipeline decreases by $40.24 \mathrm{~Hz}$ after optimization, and the $11^{\text {th }}$ natural frequency increases by $49.21 \mathrm{~Hz}$ after optimization. The calculation results of statistical candidate points meet the requirements of support optimization, which can improve the calculation time significantly.

\section{Conclusion}

This paper proposed a developed response surface method to reduce the vibration by optimizing the natural frequency of the pipeline system. The BBD scheme is presented to establish the response surface model between the design variable and the response. New results and conclusions are obtained.

The fitting degree of the sample points of the response surface between the simulation and prediction value is linear, and the predicted line is in good agreement with P4 and P5, which can verify the effectiveness of the response surface model. The response surface model shows complex nonlinear characteristics between P1, P2, P3 and P4 and P5 in order to obtain the optimal solution. The optimization results based on the response surface method can optimal the natural frequencies of pipeline significantly, which is more than $20 \%$ away from the pump excitation frequency.

In the design process of offshore pipeline system, the dynamic optimization analysis is essential to ensure that there is no harmful resonance in the working conditions. The proposed developed response surface method in this paper can not only improve the vibration performance of the hydraulic pipeline system, but also improve the calculation efficiency significantly. It provides a new practical optimization method for vibration analysis of offshore hydraulic pipeline system.

Conflict of Interest: The author declare that there is no conflict of interest regarding the publication of this paper. Acknowledgments: This work is supported by Natural Science Foundation of Shandong Province (Grant no. ZR2018MEE021) and Equipment Pre Research Fund Project (Grant no. 61402100501).

\section{References}

[1] Kwong, A.H.M.; Edge, K.A. A method to reduce noise in hydraulic systems by optimizing pipe clamp locations. Proceedings of The Institution of Mechanical Engineers Part I Journal of Systems \& Control Engineering, 1998, 212(4):267-280.

[2] Wang, D.; Jiang, J.S.; Zhang, W.H. Optimization of support positions to maximize the fundamental frequency of 
structures. International Journal for Numerical Methods in Engineering, 2004, 61(10):1584-1602.

[3] Wang, D. Optimization of support positions to minimize the maximal deflection of structures. International Journal of Solids \& Structures, 2004, 41(26):7445-7458.

[4] Liu, Y.S.; He, X.D.; Zhu, Y.H. et al. Dynamical strength and design optimization of pipe-joint system under pressure impact load. Proceedings of the Institution of Mechanical Engineers, 2012, 226(G8):1029-1040.

[5] Li, X.; Zhang, L.J.; Wang, S.P. et al. Impedance analysis and clamp locations optimization of hydraulic pipeline system in aircraft. International Conference on Fluid Power \& Mechatronics, Ha Erbin, China, 2015.08.05, pp.10231028.

[6] Gao, P.X.; Li, J.W.; Zhai, J.Y. et al. A novel optimization layout method for clamps in a pipeline system. Applied Sciences. 2020, 10(1): 390.

[7] Box G, Wilson K. On the experimental attainment of optimum condition. Journal of the Royal Statistical Society, 1951, 13(1): 1-45.

[8] Xiao, M.H.; Shen, X.J.; Liu, X. Study on properties of 45 Carbon Steel Ni-P electroless plating reinforced by Si3N4Al2O3 particle based on response surface method. Journal of Nanoscience and Nanotechnology. 2020, 20, 4761-4772.

[9] Zhang, T.; Zhou, X.P.; Liu, X.F. Reliability analysis of slopes using the improved stochastic response surface methods with multicollinearity. Engineering Geology. 2020, 271, doi: 10.1016/j.enggeo.2020.105617.

[10] Abba, S.I.; Usman A.G.; Isik, S. Simulation for response surface in the HPLC optimization method development using artificial intelligence models: A data-driven approach. Chemometrics and Intelligent Laboratory Systems. 2020,201, doi: 10.1016/j. chemolab.2020.104007.

[11] Gao, L.; Gegentana; Liu, Z.Z.; Sun. B.Z.; Li, S.H. Multiobjective optimization of thermal performance of packed bed latent heat thermal storage system based on response surface method. Renewable Energy. 2020, 153, pp. 169-180.

[12] Perwez, A.; Siddiqui, N.A.; Alqahtani, A.S.; Haque, A. Response surface methodology-based optimization of ultrasound-assisted extraction of beta-sitosterol and lupeol from astragalus atropilosus (roots) and validation by HPTLC method. Asian Pacific Journal of Tropical Biomedicine. 2020, 10, pp. 281-292.

[13] Heddam, S.; Keshtegar, B.; Kisi, O. Predicting total dissolved gas concentration on a daily scale using kriging interpolation, response surface method and artificial neural network: Case Study of Columbia River Basin Dams, USA. Natural Resources Research. 2020, 29, pp. 1801-1818.

[14] Xie, Y.W.; Hu, P.F.; Zhu, N.; Lei, F.; Xing, L.; Xu, .L.H. Collaborative optimization of ground source heat pumpradiant ceiling air conditioning system based on response surface method and NSGA-II. Renewable Energy.2020, $147,149-167$.

[15] Shirazi, M.; Khademalrasoul, A.; Ardebili, S.M.S. Multiobjective optimization of soil erosion parameters using response surface method (RSM) in the Emamzadeh watershed. Acta Geophysica.2020, 68, pp.505-517.

[16] Xue, X.F.; Wang, Y.Z.; Lu, C. Sinking velocity impactanalysis for the carrier-based aircraft using the response surface method-based improved kriging algorithm. Advances in Materials Science And Engineering. 2020, 202, 10.1155/2020/5649492.

[17] Solmaz, H.; Ardebili, S.M.S.; Aksoy, F.; Calam, A.; Yilmaz, E. Arslan. Optimization of the operating conditions of a beta-type rhombic drive stirling engine by using response surface method. Energy. 2020, 198, doi: 10.1016/j. energy.2020.117377.

[18] Bai, B.; Li, H.; Zhang, W.; Cui, Y.C. Application of extremum response surface method-based improved substructure component modal synthesis in mistuned turbine bladed disk. Journal of Sound and Vibration. 2020, 472, doi: 10.1016/j.jsv.2020.115210.

[19] Su, Y.; Fu, G. Wan, Bo. et al. Fatigue reliability design for metal dual inline packages under random vibration based on response surface method. Microelectronics Reliability, 2019, 100, 113404.

[20] Ferreira, S.; Bruns, R.; Ferreira, H. et al. Box-Behnken design: an alternative for the optimization of analytical methods. Analytica Chimica Acta, 2007, 597(2):179-186. 